\section{How it all began: a brief history of the Internet}

\section{by Alice Keefer and Tomas Baiget}

When an article on the history of the Internet was first suggested, our reaction was, "But doesn't everyone already know how it started?" Having lived the experience (or, perhaps more aptly, having survived it), we had become like veterans of any major event who assume that certain facts will always be maintained in the collective memory. However, we ourselves -from the US and Spain, respectively- have noted with incredulity the mistaken answers given by members of the younger generations among our compatriots to such questions as: "In what Southeast Asian country did the US fight a war?" or "Who was Francisco Franco?". While for some, the answers are burnt into the cerebral circuitry, the younger respondents treat the questions as so many Trivial Pursuit challenges, on the same par as "What team did Brazil beat in the 1962 World Cup?" or "What was the name of the boy actor who played Timmy in the original Lassie series?"

While the Internet's development may lack the emotional charge of the Vietnam War or the Franco dictatorship, it has had a whopping effect on the society in which we live, including commerce, finance, health, education, politics, leisure, etc. So,we accepted the charge to write a brief historical review of the Internet at a time which very neatly (albeit roughly) coincides with several significant anniversaries: approximately 30 years since the Internet's inception; 10 years since its liberalisation which opened the floodgates to new users; and 5 years since the incorporation of Web applications into the mainstream. ${ }^{1}$

We imagine the article to be addressed to two different demographic groups: 1) those who were working in the information field during the past 510 years and who have seen their professional activities (and perhaps their personal lives) altered by the Internet, and 2) those that have come into the labour market in the past 5 years, to whom the Internet has always been a "given". We hope that there will be something new for everyone. So join us for a walk, with musical accompaniment, down memory lane. ${ }^{2}$

\section{Revolution, Beginnings of}

[Authors ' note: Those readers that already know that Internet had its beginnings as a defence project may jump to the next section.]

Following the Second World War and lasting through to the late 1980's, a Cold War was fought between the world's two superpowers - the United States and the now defunct Union of Soviet Socialist Republics. While military build ups, political sparring, and diplomatic manoeuvring were the most obvious activities, there were also many spin-off effects into other areas including some nearer and dearer to the heart of librarians and documentalists, such as research and education. For example, the USSR's jump-start of the space race with the launch of the Sputnik satellite in 1957 sparked a dedicated effort in the United States to boost scientific research. Academic and research libraries, by and large, became beneficiaries of the government largesse that resulted from this "knowledge race." Collections boomed in order to keep up with the production of scientific literature. In order to control the resulting surge in bibliographical data, computers were enlisted to process the information then published as print or microfilmed indexes, and which subsequently would be transformed into databases accessible through the large host services that sprang up in the early 1970 s, such as Dialog, SDC-Orbit, ESAIRS (DialTech), Blaise, BRS, etc.

\section{Back in the US..., back in the US..., back in the USSR}

The 1960s saw several near collisions between the US and the USSR, the most notable being the Cuban missile crisis in 1962, which had Americans scrambling to build and supply bomb shelters in their suburban back yards. Although that particular crisis was defused when the USSR agreed to withdraw its nuclear projectiles from Cuba, patriotic paranoia persisted.

With this mental set as a backdrop and as computers became more prevalent in large government and research installations, the US Defense Department's Advanced Research Projects Agency (ARPA) was commissioned to establish a secure 
network linking centres working on military research in such a way that the communications would remain intact even if some of the centres were knocked out (e.g., due to an enemy attack). This strategic requirement led to the development of a distributed network, whereby -theoretically, at least - the connections would continue to work even in the absence of one or more of its parts. An initial trial between computers at UCLA and the Stanford Research Institute took place in 1969 as described below:

On the evening of October 29th, the first attempt was made to connect from UCLA to SRI, 350 miles to the north. The machines were on, the digital circuit connecting the IMPs [Interface Message Processor] was ready, each end confirmed all was "go" via a phone connection. So it began.

The idea was to first get the computers connected to each other via the IMPs, then do a little data transfer. To do the first part, the UCLA site was to login to the SRI site. With [Dr. Leonard] Kleinrock watching over his shoulder, Charley Kline typed an L. SRI confirmed receipt of the $\mathrm{L}$. He typed an $O$. SRI confirmed receipt of the $\mathrm{O}$. He typed a G. SRI confirmed a system crash! But all was not lost. In a few hours the problem was fixed, LOGIN was received, a good connection was established, and the initial transmission experiments were carried out. ${ }^{3}$

By the end of that same year, computers at the University of California Santa Barbara and the University of Utah also were connected to this pioneering venture, named ARPAnet. A year later there were ten nodes spanning the country.

At the time, one of the very novel qualities of ARPAnet was its ability to connect heterogeneous sites, since prior networking was for the most part limited to same-system equipment.

The first public demonstration of ARPAnet took place in October 1972 at an international conference on computer communications. In the words of Vinton Cerf-considered one of the "fathers" of the Internet, "The demo was a roaring success, much to the surprise of the people at AT\&T who were sceptical about whether it would work." The following year the University College of London and the Norwegian Royal Radar Establishment became the first international sites to connect to ARPAnet.

As is well known, the original applications of ARPAnet were Telnet for logging on as a local terminal to remote computers, File Transfer (FTP) for moving files between computers, and email (SMTP) for exchanging messages.

Although we may never know what military secrets were passed between secure machines, one thing is sure: the people sitting behind those machines recognised the usefulness of sending human readable messages among themselves, thus turning e-mail into the Internet's first "killer app". Although it may not have been ARPAnet's main priority, it ensured that the Internet became entrenched in the daily lives of millions of persons.

What types of messages were being exchanged by these high level techies? A glimpse into the rarefied atmosphere of early Internet sites is provided by Bob Bell, who worked at the Stanford site in the early 1970 s:

I remember hearing that there was an ARPANET "conference" on the Star Trek game every Friday night. Star Trek was a text based game where you used photon torpedos and phasers to blast Klingons. ${ }^{5}$

\section{Please Mr. Postman}

How was it achieved? The ARPAnet developed the Internet protocol (IP), whereby messages are passed along by routers -computer nodes acting as mail sorters - that determine at any given moment the best path for directing a packet to its destination. Broken links in the network can thereby be avoided. The system is based on a simple addressing scheme using the (now-ubiquitous) IP address. The IP number, for instance 111.222.333.444, begins with the code for the network on the left and works down to the number of an individual computer at the right side. Thus its structure goes from general to specific, which curiously is just the opposite of the traditional postal addressing system, which begins with the specific (name of intended recipient) and ends with the general (state/province and country).

The related "domain names" were later introduced, allowing numbers - easily retained by machines to be replaced by alphabetical codes that are more 
easily retained by human memory. As everyone knows, this is the addressing system used on the World Wide Web. The original top level domains (TLDs) were limited to: .com, .edu, .gov, .mil, .org, and .net, as well as two digit country codes. In response for growing demand, the Internet Corporation for Assigned Names and Numbers (ICANN) recently approved seven new TLDs for subsequent negotiating of agreements: aero, .biz, .coop, info, .museum, name, and .pro. <http:// www.icann.org/tlds/ $>$.

The other half of the Internet communications' dynamic duo known as TCP/IP is the Transmission Control Protocol, which establishes the means by which the messages are broken down into discrete packages for sending separately and subsequently rearranged when the parts meet up again at the destination site.

\section{This LAN is your LAN, this LAN is my LAN...}

As local area networks expanded in the $1980 \mathrm{~s}$, other institutions also adopted the IP protocol and, as more and more joined the club, they were all able to communicate amongst themselves, thus effectively creating the network of networks that was to receive the name "Internet". But perhaps the most significant shift took place in the mid1980 s when the US government, through the National Science Foundation, funded the establishment of a network of five supercomputing centres, NSFnet. As demand grew, regional centres were established, opening up functionality previously limited to highly specialised users and making it generally available to huge and diverse university populations, thus contributing to the acceptance of the IP protocol as the ad hoc standard.

The intention of NSFnet was to support research and education in US institutions. The NSF's policy declaration for the acceptable use of the Internet prohibited the following activities on its portion of the Net:

\section{Unacceptable uses - NSFnet:}

- Use for for-profit activities, unless covered by the General Principle or as a specifically acceptable use.

- Extensive use for private or personal business.
Continuing along this line, in his 1992 book, The Whole Internet, that became the bible of many first-time Internet users, Ed Krol explains the importance of self-regulation on the Net. Behaviour to be avoided at that time included:

- $\quad$ Excessive game playing;

- $\quad$ Excessive ill-conceived use;

- Hateful, harassing, or other antisocial behaviour;

- Intentional damage or interference with others;

- Publicly accessible obscene files. ${ }^{6}$

More proof-if any is needed - that the Internet does not remain static!? For an updated reality check, a recent article in The Economist states that "airline tickets rival pornography as the hottestselling commodity on the Internet" ${ }^{8}$

\section{Move over, Beethoven}

Throughout the 1980s and early 1990 s the Internet enjoyed steady, sustained growth. In 1989, Tim Berners-Lee of the European Organisation for Nuclear Research (CERN) wrote a proposal for establishing a global hypertext system with the suggested name of MESH. ${ }^{9}$ It worked its way from proposal to demonstrable prototype to full fledged system over the next several years. But the lid was finally blown off the relatively complacent evolution of the Internet when, in the mid-1990s, the World Wide Web became accessible to millions, thanks to the development of user-friendly web browsers, beginning with Mosaic. The Mosaic Communications Corp. (now Netscape), created in March 1994, was staffed by the original team of developers from the University of Illinois, who had been drafted en masse by their former colleague, Marc Andreesen.

At that point, all of the previous soul-searching about the proper uses of the Internet and reticence concerning its commercialisation, became moot issues as the existing walls set up by academic and government efforts were flattened by the incoming stampede of interests from every sector.

As of November 2000, there were calculated to be 407.1 million users, 113.14 million of which are 
estimated to Europe. Obviously, size and usage figures are quickly outdated, and so interested readers are advised to check other sites for the most current data, such as NUA <http:// www.nua.ie/surveys/>.

Since much information concerning the Internet has a US slant, it is worth highlighting some EC-funded initiatives that contribute to our knowledge of Internet use in different economic and social sectors within the member countries.

ECATT (Benchmarking Progress on Electronic Commerce and New Methods of Work) $<$ http://www.eto.org.uk/ist-nmw/present/ecatt/ sld001.htm>.

Funded under the 4th FP (ACTS and ESPRIT) this project has completed the benchmarking of ecommerce and telework developments in 10 countries.

EMERGENCE (Estimation and Mapping of Employment in a Global Economy in the New Communications Environment) $<$ http://www.emergence.nu/>.

Emergence is concerned with the relocation of work as e-business activities develop. It therefore involves substantial surveys of e-business developments in most EU countries as well as in the USA.

NEWKIND (New Indicators for the Knowledge Based Economy) <http://www.researchineurope.org/newkind/ index.htm $>$.

The project will develop indicators on the accumulation of intangible capital; e-commerce infrastructures, and "performance/efficiency gain".

ERMIS (Electronic commeRce Measurement through Intelligent agentS)

$<$ http://www.medialab.ntua.gr/Ermis/ index.htm>.

ERMIS will develop indicators of consumeroriented electronic commerce using web-based agents.

EICSTES (European Indicators, Cybermetrics and the Science-Technology-Economy System) <http://www.eicstes.org>.

This project will develop indicators on the impact of the new economy on innovation.
STING (Evaluation of Scientific \&

Technological Innovation and Progress in Europe through Patents) <http:// www.empirica.com/en/content_main.htm>. STING will develop indicators on technology innovation based on patent data.

\section{FAMILIES (Families, Work and IST) <http://www.uk.teknologisk.dk/2938>. This project will analyse impacts of work on families, associated with changes in IST work organisation.}

STAR (Socio-economic Trends Assessment for the Digital Revolution) $<$ http://www.empirica.com/en/content_main.htm>. STAR will develop indicators and analyse emerging patterns of development of the digital economy; the skills gap; new ways to work; epayments and smart card use; e-government.

\section{SIBIS (Statistical Indicators for Benchmarking the Information Society) \\ $<$ http://www.empirica.com/en/content_main.htm $>$. A follow-up to the 4th FP EcaTT project, SIBIS will continue the benchmarking of e-business and e-work take-up, reflecting e-Europe priorities.}

\section{MISSION (Multi-agent Integration of Shared Statistical Information over the (inter)Net) <http://www.epros,ed.ac.uk/mission>.}

\section{CLAMOUR (CLAssifications MOdelling and Utilities Research) \\ $<$ http://www.statistics.gov.uk/methods_quality/ clamour/default.asp $>$. \\ Methodology, tools, users' needs and practical applications: improving the quality of existing and future classification systems}

\section{DIASTASIS (Digital Era Statistical Indicators) This project is still in its infancy and had no website at the time of writing this article. It will develop indicators on usage patterns of the Internet.}

\section{'Cause I'm the taxman}

The Internet, being the base of the so-called the "New" or "Emerging Economy", has prompted interest on the part of national statistical offices, international bodies such as Eurostat, OECD, UN, etc., and other world wide economic agents. They 
are trying to determine the value of this new. commerce, new goods, new ways of selling, etc. In addition to identifying new activities, national governments are especially focussed on the potential effect of another phenomenon: the blurring of national borders. Of special concern is the absolute ease with which goods can be bought and sold on the Web, oblivious to existing borders or tax laws. This is especially true in the case of intangible commodities and services (software, images, education, sex), making these activities even harder to measure and control, to the detriment of tax revenues.

\section{A long and windy road}

Who's in charge and where are we heading? Although the strength of the Internet is often said to lie in its utter anarchy, there are some overseers to the network.

The Internet Society is the international organisation that promotes global co-operation and co-ordination for future development of the Internet. Its principal purpose, as stated on its web site, is

to maintain and extend the development and availability of the Internet and its associated technologies and applications - both as an end in itself, and as a means of enabling organizations, professions, and individuals worldwide to more effectively collaborate, cooperate, and innovate in their respective fields and interests. ${ }^{10}$

The main governing body, WorldWideWeb Consortium (W3C), created in October 1994, sees its role as leading the Web to its "full potential by developing common protocols. Its seven guiding principles are:

- Universal access;

- Semantic web;

- Trust;

- Interoperability;

- Evolvability;

- Decentralisation;

- Cooler Multimedia!"1
Some major initiatives that will determine the future of the Internet are the US government's Next Generation Internet (NGI) and the US university consortium, Internet2. Much of their attention is being focussed on setting up a faster backbone and creating a more stable networking environment, in a replay of the NSFnet objectives of the 1980s.

The NGI initiative <http://www.ngi.gov/> began October 1, 1997, and counts among its founding members several key players from the original Internet project, Defense Advanced Research Projects Agency and the National Seience Foundation, as well as the National Aeronautics and Space Administration, National Institutes of Health, and National Institute of Standards and Technology. In their own words, the participants expect to "develop advanced networking technologies, developing revolutionary applications that require advanced networking, and demonstrating these capabilities on test beds that are 100 to 1,000 times faster end-to-end than today's Internet".

Similarly, Internet $2<\mathrm{http}: / /$ www.internet2.edu $>$ involving more than 180 universities working together with industry and government, seeks to develop and deploy "advanced network applications and technologies, accelerating the creation of tomorrow's Internet".

\section{Conclusion}

A classic work directed at information professionals made predictions about the "Neoelectronic" age. ${ }^{10}$ In it the authors drew comparisons with the Neolithic era in which the development of finer tools marked a major shift over the primitive stone tools of the Mesolithic era. Their idealised vision of the future-which smacked of science fiction merely 9 years ago- is described below:

When we arrive in the Neoelectronic era, we will have developed sophisticated network agents to be sent into the network to search and retrieve data with a high level of precision and recall. High-speed bandwidths will permit greater amounts of data to be delivered and processed than ever previously conceived.

...multimedia information will be available from a wide variety of sources and will be 
easily processed using powerful computing tools in our homes, our offices, and in our transportation systems. As the tools become more refined, more timely, and more cost effective, the virtual space will begin to support a greater number of individuals. The wide availability of information in the market will lead to a new age of enlightenment. [The emphasis is ours AK; TB]

Sounds familiar - up until the end - for if we are not yet there, we are certainly close. However, the final prediction of the book's authors has not been realised as yet, and may not be reachable in our lifetimes, if ever. Who knows? After all, the answer is blowin' in the WWWind.

\section{References}

1. For fuller histories, the interested reader can find references at the Internet Society website. <http://www.isoc.org/internet/ history/>.

2. We provide the title, you provide the tune!

3. De Violini, Robert. "Sescal 99 Notes the Start of the Internet at UCLA". <http:// www.sescal.org/inetstory.htm>.

4. Cerf, Vinton. "How the Internet Came to Be," as told to Bernard Aboba. c1993. $<$ http://www.netvalley.com/archives/ mirrors/cerf-how-inet.txt>.

5. As quoted in : Gromov, Gregory R. History of Internet and $W W W$ : The Roads and
Crossroads of Internet History $<$ http:// www.netvalley.com/intvall.html>.

6. Krol, Ed. The Whole Internet User's Guide \& Catalog. Sebastopol, CA: O'Reilly \& Associates, Inc., 1992.

7. As quoted in : Gromov, Gregory R. History of Internet and WWW: The Roads and Crossroads of Internet History <http:// www.netvalley.com/intvall.html>.

8. "Britain takes to the air," The Economist. (30 June, 2001) 32.

9. Berners-Lee, Tim. Information Management: A Proposal. March 1989. $<$ http://www.w3.org/History/1989/ proposal.html>.

10. Internet Society. <http://www.isoc.org/isoc/ general $/>$.

11. World Wide Web Consortium. http:// www.w3.org>.

12. Lane, Elizabeth; Summerhill, Craig. Internet Primer for Information Professionals: a basic guide to internet networking technology. Westport: Mecklermedia, 1993.

\section{Contact Details}

Alice Keefer

Facultat de Biblioteconomia i Documentació, Universitat de Barcelona

Email: akeefer@arrakis.es

Tomas Baiget

Institut d'Estadística de Catalunya

Email: baiget@sarenet.es 